\title{
Partition-theoretic formulas for arithmetic densities, II
}

\author{
Ken Ono, Robert Schneider and Ian Wagner
}

In honour of Srinivasa Ramanujan on the 100th anniversary of his passing

\begin{abstract}
In earlier work generalizing a 1977 theorem of Alladi, the authors proved a partition-theoretic formula to compute arithmetic densities of certain subsets of the positive integers $\mathbb{N}$ as limiting values of $q$-series as $q \rightarrow \zeta$ a root of unity (instead of using the usual Dirichlet series to compute densities), replacing multiplicative structures of $\mathbb{N}$ by analogous structures in the integer partitions $\mathcal{P}$. In recent work, Wang obtains a wide generalization of Alladi's original theorem, in which arithmetic densities of subsets of prime numbers are computed as values of Dirichlet series arising from Dirichlet convolutions. Here the authors prove that Wang's extension has a partition-theoretic analogue as well, yielding new $q$-series density formulas for any subset of $\mathbb{N}$. To do so, we outline a theory of $q$-series density calculations from first principles, based on a statistic we call the " $q$-density" of a given subset. This theory in turn yields infinite families of further formulas for arithmetic densities.
\end{abstract}

Keywords. integer partitions; arithmetic density; q-series; q-binomial theorem

2010 Mathematics Subject Classification. 05A17, 11P82

\section{Introduction and statement of results}

It is well-known that the Möbius function satisfies

$$
-\lim _{N \rightarrow \infty} \sum_{n=2}^{N} \frac{\mu(n)}{n}=1 .
$$

This fact can be used to obtain formulas for arithmetic densities for suitable subsets of the positive integers. A celebrated example of this idea was obtained by Alladi [Al77], who proved for $r, t, \in \mathbb{N}$ that if $\operatorname{gcd}(r, t)=1$, then

$$
-\sum_{\substack{n \geq 2 \\ p_{\min }(n) \equiv r}} \frac{\mu(n)}{n}=\frac{1}{\varphi(t)} .
$$

Here $\varphi(t)$ is Euler's phi function, and $p_{\min }(n)$ is the smallest prime factor of $n$.

Over the last few years, (1.2) has been generalized in various ways in works by Dawsey [Da17], Sweeting and Woo [SwWo19], Kural, McDonald and Sah [KMS], and Wang [Wa1, Wa2]. In a recent preprint [Wa3], Wang obtained a beautiful generalization of this phenomenon which makes use of Dirichlet convolutions. If $a: \mathbb{N} \rightarrow \mathbb{C}$ is an arithmetic function satisfying $a(1)=1$ and $\sum_{n \geq 2} \frac{|a(n)|}{n} \log \log n<\infty$, then for suitable subsets $\mathcal{S}$ of the positive integers Wang proves that

$$
-\lim _{N \rightarrow \infty} \sum_{\substack{2 \leq n \leq N \\ p_{\min }(n) \in \mathcal{S}}} \frac{(\mu * a)(n)}{n}=d_{\mathcal{S}},
$$

where $(f * g)(n)=\sum_{d \mid n} f(d) g(n / d)$ is the classical Dirichlet convolution of $f$ and $g$.

The first author thanks the support of the Thomas Jefferson Fund and the NSF (DMS-1601306 and DMS-2002265).

We thank episciences.org for providing open access hosting of the electronic journal Hardy-Ramanujan Journal 
In earlier work the authors obtained a partition-theoretic analogue of formulas such as (1.2) using natural analogies between the multiplicative structure of the integers and the additive structure of partitions. The purpose of this note is to show that an analogue also exists for (1.3), as a consequence of a general theorem offering infinitely many formulas (see Theorem 1.3).

We first recall fundamental features of these analogies. A partition is a finite non-increasing sequence of positive integers, say $\lambda=\left(\lambda_{1}, \lambda_{2}, \ldots, \lambda_{\ell(\lambda)}\right)$, where $\ell(\lambda)$ denotes the length (number of parts) of $\lambda$. The size of $\lambda$ is $|\lambda|:=\lambda_{1}+\lambda_{2}+\cdots+\lambda_{\ell(\lambda)}$ (sum of parts) and the norm of the partition is $N(\lambda)=\lambda_{1} \cdot \lambda_{2} \cdots \lambda_{\ell(\lambda)}$ (product of parts). ${ }^{1}$ Furthermore, we let $\operatorname{sm}(\lambda):=\lambda_{\ell(\lambda)}$ denote the smallest part of $\lambda$ (resp. $\lg (\lambda):=\lambda_{1}$ the largest part of $\lambda$ ).

We will use the partition Möbius function

$$
\mu_{\mathcal{P}}(\lambda):= \begin{cases}0 & \text { if } \lambda \text { has repeated parts } \\ (-1)^{\ell(\lambda)} & \text { otherwise. }\end{cases}
$$

Notice that $\mu_{\mathcal{P}}(\lambda)=0$ if $\lambda$ has any repeated parts, which is analogous to the vanishing of $\mu(n)$ for integers $n$ which are not square-free. In particular, the different parts in a partition $\lambda$ play the role of prime factors of $n$ in this analogy. We define $\mu_{\mathcal{P}}^{*}(\lambda):=-\mu_{\mathcal{P}}(\lambda)$ as in Dawsey's theorem, to eliminate minus signs in our formulas. Finally, we recall the usual $q$-Pochhammer symbol $(a ; q)_{n}:=$ $\prod_{k=0}^{n-1}\left(1-a q^{k}\right)$ and $(a ; q)_{\infty}:=\lim _{n \rightarrow \infty}(a ; q)_{n}$ for $a, q \in \mathbb{C},|q|<1$.

To complete the analogy, also recall the partition phi function defined in [Sc17], an analogue of Euler's totient function,

$$
\varphi_{\mathcal{P}}(\lambda):=N(\lambda) \cdot \prod_{\substack{k \in \lambda \\ \text { without repetition }}}\left(1-k^{-1}\right),
$$

where " $k \in \lambda$ " means $k \in \mathbb{N}$ is a part of $\lambda \in \mathcal{P}$.

Table 1 offers some of the identifications making up this multiplicative-additive analogy.

\begin{tabular}{|c|c|}
\hline Natural number $n$ & Partition $\lambda$ \\
\hline Prime factors of $n$ & Parts of $\lambda$ \\
\hline Square-free integers & Partitions into distinct parts \\
\hline$\mu(n)$ & $\mu_{\mathcal{P}}(\lambda)$ \\
\hline$\varphi(n)$ & $\varphi_{\mathcal{P}}(\lambda)$ \\
\hline$p_{\min }(n)$ & $\operatorname{sm}(\lambda)$ \\
\hline$p_{\max }(n)$ & $\lg (\lambda)$ \\
\hline$n^{-s}$ & $q^{|\lambda|}$ \\
\hline$\zeta(s)^{-1}$ & $(q ; q)_{\infty}$ \\
\hline$s \rightarrow 1$ & $q \rightarrow 1$ \\
\hline
\end{tabular}

Table 1: Analogies between arithmetic and partition theory

Remark 1.1. We note that other, different multiplicative-additive analogies exist, such as those drawn in [SCSi20] between partitions of length $m$ and those of size $m$, in which the duality instead correlates $\zeta(s)$ to the geometric series $q(1-q)^{-1}$, with $m^{-s}$ in bijection with $q^{m}$.

To describe our results, let $\mathcal{S}$ be a subset of the positive integers with arithmetic density

$$
d_{\mathcal{S}}:=\lim _{N \rightarrow \infty} \frac{\#\{n \in \mathcal{S}: n \leq N\}}{N} .
$$

\footnotetext{
${ }^{1}$ The norm is referred to as the "integer" of $\lambda$ and written $n_{\lambda}$ in some earlier works [ORS17, Sc16].
} 
As noted above, we let $\mu_{\mathcal{P}}^{*}(\lambda):=-\mu_{\mathcal{P}}(\lambda)$. In [OSW17] the function

$$
F_{\mathcal{S}}(q):=\sum_{\substack{\lambda \in \mathcal{P} \\ \operatorname{sm}(\lambda) \in \mathcal{S}}} \mu_{\mathcal{P}}^{*}(\lambda) q^{|\lambda|}
$$

was defined, and it was shown, for suitable $\mathcal{S}$ and associated roots of unity $\zeta$, that

$$
\lim _{q \rightarrow \zeta} F_{\mathcal{S}}(q)=d_{\mathcal{S}}
$$

This theorem is a partition-theoretic analogue of (1.2).

Remark 1.2. We note that throughout this paper, " $q \rightarrow \zeta$ " with $\zeta$ a root of unity (including the case $\zeta=1)$ denotes that $q$ approaches the point on the unit circle from within the unit disk.

To state the partition analogue of Wang's theorem requires some further notation for partition multiplication, division, and subpartitions that are analogous to partition divisors. For two partitions $\lambda, \delta \in \mathcal{P}$, we define $\delta \lambda$ to be the partition obtained by concatenating the parts of $\lambda$ to $\delta$ (and then reordering by size by notational convention). We write $\delta \mid \lambda$ if all of the parts of $\delta$ appear in the partition $\lambda$ with greater than or equal multiplicities and define the subpartition $\lambda / \delta$ as the partition obtained by removing those parts, counting multiplicities, from $\lambda$.

For two functions $f$ and $g$ on partitions, we define their partition Dirichlet convolution by

$$
(f * g)(\lambda):=\sum_{\delta \mid \lambda} f(\delta) g(\lambda / \delta)
$$

where the sum is taken over the subpartitions $\delta$ of $\lambda$ (including the empty partition $\delta=\emptyset$ ).

To state our results succinctly, we define an auxiliary series that effectively serves to identify different classes of density results. For $|q|<1, a(\lambda)$ a function on $\mathcal{P}$, and $n \geq 1$, let

$$
A_{n}(q):=a((n))+\sum_{\operatorname{sm}(\gamma) \geq^{*} n}[a(\gamma \cdot(n))-a(\gamma)] q^{|\gamma|}
$$

with $(n) \in \mathcal{P}$ the partition of $n$ with one part, $\gamma \cdot(n) \in \mathcal{P}$ the partition formed by adjoining part $n$ to partition $\gamma$, and with $\geq^{*}$ denoting $>$ if $a(\lambda)=0$ at partitions with any part repeated, and denoting $\geq$ otherwise.

We introduce one final piece of terminology: throughout this paper, the formulas we prove hold true for "nice" subsets $\mathcal{S} \subseteq \mathbb{N}$ amenable to $q$-series density calculations, which we call $q$-commensurate subsets (see Section 3 below for the precise definition).

Theorem 1.3. Let $\mathcal{S} \subseteq \mathbb{N}$ be a q-commensurate subset, and $a(\lambda)$ a function on $\mathcal{P}$ such that $a(\emptyset)=1$. If $\lim _{q \rightarrow 1} A_{n}(q)=1$ when $n \in \mathcal{S}$, then

$$
\lim _{q \rightarrow 1} \sum_{\operatorname{sm}(\lambda) \in \mathcal{S}}\left(\mu_{\mathcal{P}} * a\right)(\lambda) q^{|\lambda|}=0 .
$$

If $\lim _{q \rightarrow 1} A_{n}(q)=1+f(n)$ for $f(n)$ an arithmetic function nonzero when $n \in \mathcal{S}$, then

$$
\lim _{q \rightarrow 1} \sum_{\operatorname{sm}(\lambda) \in \mathcal{S}} \frac{\left(\mu_{\mathcal{P}} * a\right)(\lambda)}{f(\operatorname{sm}(\lambda))} q^{|\lambda|}=d_{\mathcal{S}}
$$

If we set $f(n)=n$, Theorem 1.3 gives the partition analogue of (1.3). Immediately we have a nice generalization of formula (1.6) with $\zeta=1$. 
Corollary 1.4. For $\mathcal{S} \subseteq \mathbb{N}$ a q-commensurate subset, if $\lim _{q \rightarrow 1} A_{n}(q)=0$ when $n \in \mathcal{S}$, then

$$
-\lim _{q \rightarrow 1} \sum_{\operatorname{sm}(\lambda) \in \mathcal{S}}\left(\mu_{\mathcal{P}} * a\right)(\lambda) q^{|\lambda|}=d_{\mathcal{S}}
$$

Remark 1.5. Set $a(\lambda)=\sum_{\delta \mid \lambda} \mu_{\mathcal{P}}(\delta)=1$ if $\lambda=\emptyset$ and $=0$ otherwise; one computes $A_{n}(q)=0$ for all $n \geq 1$. Then the theorem reduces to the main result of [OSW17], viz. $\lim _{q \rightarrow 1} F_{\mathcal{S}}(q)=d_{\mathcal{S}}$.

Furthermore, for an arithmetic function $a(n)$, Wang defines $b(n):=\sum_{d \mid n}(\mu * a)(d) \frac{d}{\varphi(d)}$ with $\varphi(k)$ the classical Euler phi function, and in equation (36) of [Wa3] uses Möbius inversion to show

$$
\frac{(\mu * a)(n)}{\varphi(n)}=\frac{(\mu * b)(n)}{n} \text {. }
$$

This along with the main theorem (1.3) allows Wang to prove beautiful formulas like

$$
-\lim _{N \rightarrow \infty} \sum_{\substack{2 \leq n \leq N \\ p_{\min }(n) \in \mathcal{S}}} \frac{(\mu * a)(n)}{\varphi(n)}=d_{\mathcal{S}} .
$$

Replacing $a, b$ with functions on partitions, Theorem 1.3 yields an analogue of these equations.

Corollary 1.6. Let $\mathcal{S} \subseteq \mathbb{N}$ be a q-commensurate subset, a $(\lambda)$ a function on $\mathcal{P}$ such that $a(\emptyset)=1, f$ and $g$ functions on $\mathbb{Z}_{\geq 0}$ such that $f(n) \neq 0, g(n)$ nonzero when $n \in \mathcal{S}$, and

$$
b(\lambda)=b_{a, f, g}(\lambda):=\sum_{\delta \mid \lambda}\left(\mu_{\mathcal{P}} * a\right)(\delta) \frac{g(\operatorname{sm}(\delta))}{f(\operatorname{sm}(\delta))} .
$$

If $\lim _{q \rightarrow 1} A_{n}(q)=1+f(n)$ for $n \in \mathcal{S}$, then

$$
\lim _{q \rightarrow 1} \sum_{\operatorname{sm}(\lambda) \in \mathcal{S}} \frac{\left(\mu_{\mathcal{P}} * b\right)(\lambda)}{g(\operatorname{sm}(\lambda))} q^{|\lambda|}=d_{\mathcal{S}}
$$

A version of Corollary 1.6 gives an analogue to Wang's identity (1.10).

Corollary 1.7. Let $\mathcal{S} \subseteq \mathbb{N}$ be a q-commensurate subset, and take $a(\lambda), b(\lambda)=b_{a, f, g}(\lambda)$ as in Corollary 1.6. In analogy with (1.8), define

$$
B_{n}(q):=b((n))+\sum_{\operatorname{sm}(\gamma) \geq^{*} n}[b(\gamma \cdot(n))-b(\gamma)] q^{|\gamma|} .
$$

Let $f(n)=-\varphi(n), g(n)=-1$, so that $b=b_{a,-\varphi,-1}$. If $\lim _{q \rightarrow 1} B_{n}(q)=0$ for $n \in \mathcal{S}$, then

$$
-\lim _{q \rightarrow 1} \sum_{\operatorname{sm}(\lambda) \in \mathcal{S}} \frac{\left(\mu_{\mathcal{P}} * a\right)(\lambda)}{\varphi(\operatorname{sm}(\lambda))} q^{|\lambda|}=d_{\mathcal{S}}
$$

where $\varphi(n)$ is the classical phi function.

We prove these limiting formulas in Section 4.

Remark 1.8. As noted in Section 3 below, by a Tauberian theorem of Frobenius [Fr1880], the formulas in this paper hold for every subset $\mathcal{S} \subseteq \mathbb{N}$ if one lets $q \rightarrow 1^{-}$strictly radially. 


\section{Conceptual background}

\section{A. Partition analogue of multiplicative number theory}

Before proceeding to prove the propositions in Section 1 let us discuss the multiplicative-additive confluence that provides the context in which results like these - displaying a fusion of partitiontheoretic objects with familiar-looking forms from classical multiplicative number theory — arise naturally.

In the preceding section we introduced the multiplicative partition norm $N(\lambda)$, the partition product (concatenation), partition division (deleting parts) and subpartition sums that act like sums over divisors, as well as partition analogues of classical arithmetic functions and Dirichlet convolution; this multiplicative theory of additive partitions was introduced in [Sc16, Sc17, Sc18] and subsequent work [ORS17, OSW17, ScSi20]. Some of these analogies, pertinent to this paper, are captured in Table 1.

These partition-theoretic structures behave almost identically to their classical counterparts. For instance, one can prove familiar-looking "divisor sum" identities like

$$
\begin{aligned}
& \sum_{\delta \mid \lambda} \mu_{\mathcal{P}}(\delta)= \begin{cases}1 & \text { if } \lambda=\emptyset \\
0 & \text { otherwise }\end{cases} \\
& \sum_{\delta \mid \lambda} \varphi_{\mathcal{P}}(\delta)=N(\lambda)
\end{aligned}
$$

the partition Möbius inversion formula

$$
A(\lambda)=\sum_{\delta \mid \lambda} a(\delta) \Longleftrightarrow a(\lambda)=\sum_{\delta \mid \lambda} A(\delta) \mu_{\mathcal{P}}(\lambda / \delta),
$$

and closely connected order-of-summation-swapping principle

$$
\sum_{\lambda \in \mathcal{P}} a(\lambda) \sum_{\delta \mid \lambda} b(\delta)=\sum_{\lambda \in \mathcal{P}} b(\lambda) \sum_{\gamma \in \mathcal{P}} a(\gamma \lambda)
$$

and other analogues of classical identities, like a partition Cauchy product formula for absolutely convergent series

$$
\left(\sum_{\lambda \in \mathcal{P}} a(\lambda)\right)\left(\sum_{\lambda \in \mathcal{P}} b(\lambda)\right)=\sum_{\lambda \in \mathcal{P}} \sum_{\delta \mid \lambda} a(\delta) b(\lambda / \delta),
$$

which is obviously related to partition convolution defined in (1.7). ${ }^{2}$ We note two useful consequences of (2.12) from [Sc17], setting $b=\mu_{\mathcal{P}}$ :

$$
\begin{aligned}
(q ; q)_{\infty}^{-1} \sum_{\lambda \in \mathcal{P}} a(\lambda) q^{|\lambda|} & =\sum_{\lambda \in \mathcal{P}} q^{|\lambda|} \sum_{\delta \mid \lambda} a(\delta), \\
(q ; q)_{\infty} \sum_{\lambda \in \mathcal{P}} a(\lambda) q^{|\lambda|} & =\sum_{\lambda \in \mathcal{P}} q^{|\lambda|} \sum_{\delta \mid \lambda} a(\delta) \mu_{\mathcal{P}}(\lambda / \delta) .
\end{aligned}
$$

These are $q$-series analogues of the classical Dirichlet series identities (see Table 1)

$$
\begin{aligned}
\zeta(s)^{-1} \sum_{n=1}^{\infty} f(n) n^{-s} & =\sum_{n=1}^{\infty} n^{-s} \sum_{d \mid n} f(d), \\
\zeta(s) \sum_{n=1}^{\infty} f(n) n^{-s} & =\sum_{n=1}^{\infty} n^{-s} \sum_{d \mid n} f(d) \mu(n / d) .
\end{aligned}
$$

\footnotetext{
${ }^{2}$ We note that the partition convolution operation was initially suggested to the second author by O. Beckwith (personal communication, 2018).
} 
This multiplicative-additive theory also contains broad classes of partition zeta functions

$$
\zeta_{\mathcal{P}^{\prime}}(s):=\sum_{\lambda \in \mathcal{P}^{\prime}} N(\lambda)^{-s} \quad(\operatorname{Re}(s)>1)
$$

where $P^{\prime} \subsetneq \mathcal{P}$, that yield beautiful evaluations [Sc16] and interesting analytic information [ORS17, ScSi20]. There also exist partition Dirichlet series [ORS17, Sc18] that enjoy identities generalizing classical Dirichlet series relations, e.g. if $\mathcal{P}_{\mathcal{S}}$ denotes partitions with all parts in any $\mathcal{S} \subset \mathbb{N}$,

$$
\begin{aligned}
& \sum_{\lambda \in \mathcal{P}_{\mathcal{S}}} \mu_{\mathcal{P}}(\lambda) N(\lambda)^{-s}=\frac{1}{\zeta_{\mathcal{P}_{\mathcal{S}}}(s)} \quad(\operatorname{Re}(s)>1), \\
& \sum_{\lambda \in \mathcal{P}_{\mathcal{S}}} \varphi_{\mathcal{P}}(\lambda) N(\lambda)^{-s}=\frac{\zeta_{\mathcal{P}_{\mathcal{S}}}(s-1)}{\zeta_{\mathcal{P}_{\mathcal{S}}}(s)} \quad(\operatorname{Re}(s)>2) .
\end{aligned}
$$

Taking $\mathcal{S}=\mathbb{P}$ regains the classical cases. While this is indeed looking a lot like multiplicative number theory, the same superstructure encompasses $q$-hypergeometric series, partition bijections and other aspects of additive number theory, and recovers classical theorems in those areas as well (see [Sc18] for further reading).

\section{Computing arithmetic densities with $q$-series}

\section{A. The $q$-density statistic}

For $|q|<1$, we recall that the function $F_{\mathcal{S}}(q)$ from (1.5) has two natural representations, one involving $\operatorname{sm}(\lambda)$ and one involving $\lg (\lambda)$, the smallest and largest parts of partition $\lambda$, respectively. Namely, we have

$$
F_{\mathcal{S}}(q):=\sum_{\operatorname{sm}(\lambda) \in \mathcal{S}} \mu_{\mathcal{P}}^{*}(\lambda) q^{|\lambda|}=\frac{\sum_{\lg (\lambda) \in \mathcal{S}} q^{|\lambda|}}{\sum_{\lambda \in \mathcal{P}} q^{|\lambda|}}=(q ; q)_{\infty} \sum_{n \in \mathcal{S}} \frac{q^{n}}{(q ; q)_{n}}
$$

noting $\operatorname{sm}(\emptyset)=\lg (\emptyset):=0$. We proved in [OSW17] that $\lim _{q \rightarrow 1} F_{\mathcal{S}}(q)=d_{\mathcal{S}}$ for special subsets $\mathcal{S} \subseteq \mathbb{N}$.

The story arc represented in the works of Alladi [Al77], Dawsey [Da17], Wang [Wa1, Wa2, Wa3], et al., together with our own work in [OSW17], stems from the observation that (1.1) can be interpreted as a statement about arithmetic density. Now, this statement itself can be interpreted via L'Hospital's rule and Lambert series as a statement about $q$-series, since

$$
\sum_{n \geq 2} \frac{\mu(n)}{n}=\lim _{q \rightarrow 1}(1-q)\left(\sum_{n \geq 1} \frac{\mu(n) q^{n}}{1-q^{n}}-\frac{\mu(1) q}{1-q}\right)=\lim _{q \rightarrow 1}(1-q)\left(q-\frac{q}{1-q}\right)=-1
$$

assuming the implicit order-of-limits swap with " $\lim _{q \rightarrow 1}$ " and the infinite series is valid.

The classical $q$-binomial theorem was at the heart of our results in [OSW17]. In order to prove the main results of this paper involving less familiar-looking forms, we sketch a theory of $q$-series computations of arithmetic density from first principles, based on a simple statistic.

Definition 3.1. For $|q|<1$ we define the $q$-density of $\mathcal{S} \subseteq \mathbb{N}$ by the quotient

$$
d_{\mathcal{S}}(q):=\frac{\sum_{n \in \mathcal{S}} q^{n}}{\sum_{n \geq 0} q^{n}}=(1-q) \sum_{n \in \mathcal{S}} q^{n}
$$


The $q$-density is a geometric series analogue of (3.13). ${ }^{3}$ Of course, from both the shape of $d_{\mathcal{S}}(q)$ and known instances of $F_{\mathcal{S}}(q)$, one wants to see the limiting behavior

$$
\lim _{q \rightarrow 1} \frac{\sum_{n \in \mathcal{S}} q^{n}}{\sum_{n \geq 0} q^{n}}=\lim _{N \rightarrow \infty} \frac{\sum_{n \in \mathcal{S}(N)} 1}{\sum_{0 \leq n<N} 1}=: d_{\mathcal{S}},
$$

where $\mathcal{S}(N)=\{n \in \mathcal{S}: n \leq N\}$. It is a consequence of a Tauberian theorem due to Frobenius with a converse by Hardy-Littlewood (see [Fr1880, Ko]), that in fact (3.15) holds for every subset $\mathcal{S} \subseteq \mathbb{N}$ if $q \rightarrow 1$ radially. ${ }^{4}$ In [OSW17], we prove arithmetic density formulas using other paths, and approaching other roots of unity; we do not rigorously prove general limiting properties here (certainly such a study would be useful), but side-step the issue somewhat circularly by defining it to be a property of a subset of $\mathbb{N}$ that the limit computations we undertake are valid.

Definition 3.2. We define a subset $\mathcal{S} \subseteq \mathbb{N}$ to be q-commensurate if $\lim _{q \rightarrow 1} d_{\mathcal{S}}(q)=d_{\mathcal{S}}$ is approached sufficiently rapidly for the asymptotic proofs we employ here to hold.

Remark 3.3. In full generality, a subset $\mathcal{S}$ being q-commensurate is equivalent to one's being able, for that subset, to interchange order of limits in the calculation $d_{\mathcal{S}}:=\lim _{N \rightarrow \infty} \lim _{q \rightarrow 1} \frac{\sum_{n \in \mathcal{S}(N)} q^{n}}{\sum_{0 \leq n<N} q^{n}}$, and to having all implicit error terms in the following asymptotic equalities of lower order than the main terms, so the errors vanish when multiplied by $(q ; q)_{\infty}$ as $q \rightarrow 1$.

Immediately we see that $\mathcal{S}=\mathbb{N}$ is $q$-commensurate since $d_{\mathbb{N}}(q)=q \rightarrow 1=d_{\mathbb{N}}$. The analogy between $d_{\mathcal{S}}(q)$ and $F_{\mathcal{S}}(q)$ holds quite closely with respect to density.

Theorem 3.4. Let $r \in \mathbb{Z}, t \in \mathbb{N}$. For subsets $\mathcal{S}_{r, t}:=\{n \geq 0: n \equiv r(\bmod t)\}$ we have that

$$
\lim _{q \rightarrow 1} d_{\mathcal{S}_{r, t}}(q)=d_{\mathcal{S}_{r, t}}=\frac{1}{t}
$$

Proof. The claimed limit follows from geometric series and L'Hospital's rule:

$$
\lim _{q \rightarrow 1} d_{\mathcal{S}_{r, t}}(q)=\lim _{q \rightarrow 1}(1-q) \sum_{k \geq 0} q^{k t+r}=\lim _{q \rightarrow 1} \frac{q^{r}(1-q)}{1-q^{t}}=\frac{1}{t} .
$$

Theorem 3.4 is a geometric series version of Theorem 1.3 in [OSW17]. There is also a $q$-density analogue of Corollary 1.5 in [OSW17].

Corollary 3.5. For subsets $\mathcal{S}_{\mathrm{fr}}^{(k)} \subset \mathbb{N}$ of $k$ th power-free integers, $k \geq 2$, we have that

$$
\lim _{q \rightarrow 1} d_{\mathcal{S}_{\mathrm{fr}}^{(k)}}(q)=d_{\mathcal{S}_{\mathrm{fr}}^{(k)}}=\frac{1}{\zeta(k)}
$$

Proof. The steps of the proof of Cor. 1.5 in [OSW17] hold identically here, only one replaces $F_{\mathcal{S}_{r, M}}(q)$ with $d_{\mathcal{S}_{r, M}}(q)$, then uses Theorem 3.4 in place of Th. 1.3 of [OSW17].

Thus the subsets $\mathcal{S}_{r, t}$ and $\mathcal{S}_{\mathrm{fr}}^{(k)}$ are $q$-commensurate. Table 2 illustrates the $k=2$ (square-free) case of Corollary 3.5 as $q \rightarrow 1$ radially. ${ }^{5}$

\footnotetext{
${ }^{3}$ For $q \in[0,1)$, equation (3.14) can be interpreted as the expected value of the indicator function for the subset $\mathcal{S}$, given the probability function $P(n)=q^{n-1}(1-q)$ for random $n \in \mathbb{N}$.

${ }^{4}$ Indeed, (3.15) holds if $q \rightarrow 1$ in a "Stolz sector" of the unit circle; for a somewhat more general context, the reader is referred to the second author's study [Sc], following up on the present work.

${ }^{5}$ The values in Table 2 were computed by A. V. Sills in Mathematica.
} 


\begin{tabular}{|c|c|}
\hline$q$ & $d_{\mathcal{S}_{\mathrm{fr}}^{(2)}}(q)$ \\
\hline 0.90 & $0.739971 \ldots$ \\
\hline 0.91 & $0.729108 \ldots$ \\
\hline 0.92 & $0.717828 \ldots$ \\
\hline 0.93 & $0.706100 \ldots$ \\
\hline 0.94 & $0.693895 \ldots$ \\
\hline 0.95 & $0.681180 \ldots$ \\
\hline 0.96 & $0.667921 \ldots$ \\
\hline 0.97 & $0.654062 \ldots$ \\
\hline 0.98 & $0.639523 \ldots$ \\
\hline 0.99 & $0.624215 \ldots$ \\
\hline 0.999 & $0.609613 \ldots$ \\
\hline 0.9999 & $0.608069 \ldots$ \\
\hline
\end{tabular}

Table 2: Illustration of $d_{\mathcal{S}_{f r}^{(2)}}(q)$ approaching $\frac{6}{\pi^{2}}=0.607927 \ldots$

\section{B. Further $q$-series density formulas}

The $q$-density statistic can be used as a building block for further arithmetic density relations. ${ }^{6}$ For $\mathcal{S} \subseteq \mathbb{N}$ a $q$-commensurate subset ${ }^{7}$, as $q \rightarrow 1$ it follows from (3.15) that

$$
\sum_{n \in \mathcal{S}} q^{n} \sim d_{\mathcal{S}} \cdot \frac{q}{1-q} .
$$

Because geometric series are central components of partition generating functions and $q$-series, the $q$-density may be embedded in diverse partition relations. For example, one can prove the central claims of [OSW17] (with a sufficient condition) by applying generating function methods to (3.16).

Theorem 3.6. For $\mathcal{S} \subseteq \mathbb{N}$ a q-commensurate subset, we have

$$
\lim _{q \rightarrow 1} F_{\mathcal{S}}(q)=-\lim _{q \rightarrow 1} \sum_{n \in \mathcal{S}} \sum_{k \geq 1} \frac{(-1)^{k} q^{n k+\frac{k(k-1)}{2}}}{(q ; q)_{k-1}}=d_{\mathcal{S}} .
$$

Proof. Take $q \mapsto q^{k}$ in (3.16). Multiply through by $(-1)^{k} q^{\frac{k(k-1)}{2}}(q ; q)_{k-1}^{-1}$ and sum both sides over $k \geq 1$, swapping order of summation on the left to give

$$
\sum_{n \in \mathcal{S}} \sum_{k \geq 1} \frac{(-1)^{k} q^{n k+\frac{k(k-1)}{2}}}{(q ; q)_{k-1}}
$$

For each $k \geq 1$, the factor $(q ; q)_{k-1}^{-1}$ generates partitions with largest part strictly $<k$. The factor $q^{n k}$ adjoins a largest part $k$ with multiplicity $n \in \mathcal{S}$ to each partition. The $q^{k(k-1) / 2}=q^{1+2+3+\ldots+(k-1)}$ factor guarantees at least one part of each size $<k$. Thus (3.17) is the generating function for partitions $\gamma$ with largest part having multiplicity in $\mathcal{S}$, and with every natural number $<\lg (\gamma)$ appearing as a part, weighted by $(-1)^{\lg (\gamma)}=(-1)^{k}$. Under conjugation, this set of partitions $\gamma$ maps to partitions $\lambda$ into distinct parts with smallest part $\operatorname{sm}(\lambda) \in \mathcal{S}$, weighted by $\mu_{\mathcal{P}}(\lambda)=(-1)^{\ell(\lambda)}=(-1)^{k}$ which is nonzero since $\lambda$ has no repeated part. Multiplying by -1 gives $\mu_{\mathcal{P}}^{*}(\lambda):=-\mu_{\mathcal{P}}(\lambda)$; thus

$$
-\sum_{n \in \mathcal{S}} \sum_{k \geq 1} \frac{(-1)^{k} q^{n k+\frac{k(k-1)}{2}}}{(q ; q)_{k-1}}=\sum_{\operatorname{sm}(\lambda) \in \mathcal{S}} \mu_{\mathcal{P}}^{*}(\lambda) q^{|\lambda|}=F_{\mathcal{S}}(q) .
$$

\footnotetext{
${ }^{6}$ E.g. see Section 5 . below.

${ }^{7}$ Or any subset of $\mathbb{N}$ if $q \rightarrow 1$ radially, by the result of Frobenius cited above.
} 
Manipulating the right-hand side of (3.16) accordingly, we then have

$$
-\sum_{n \in \mathcal{S}} \sum_{k \geq 1} \frac{(-1)^{k} q^{n k+\frac{k(k-1)}{2}}}{(q ; q)_{k-1}} \sim-d_{\mathcal{S}} \cdot \sum_{k \geq 1} \frac{(-1)^{k} q^{\frac{k(k+1)}{2}}}{(q ; q)_{k}}=-d_{\mathcal{S}} \cdot\left((q ; q)_{\infty}-1\right),
$$

using an identity of Euler in the last step, which clearly approaches $d_{\mathcal{S}}$ as $q \rightarrow 1$.

We don't necessarily need to restrict smallest parts to $\mathcal{S}$ to give formulas for $d_{\mathcal{S}}$. For instance, by similar methods, an analogous formula extends to the "largest parts" case.

Theorem 3.7. For $\mathcal{S} \subseteq \mathbb{N}$ a q-commensurate subset, we have

$$
\lim _{q \rightarrow 1} \sum_{\lg (\lambda) \in \mathcal{S}} \mu_{\mathcal{P}}^{*}(\lambda) q^{|\lambda|}=d_{\mathcal{S}}
$$

Proof. Take $q \mapsto q^{k}$ in (3.16). Multiply both sides by $(q ; q)_{k-1}^{-1}$, sum over $k \geq 1$, then swap order of summation and make the change of indices $k \mapsto k+1$ on the left side, to give

$$
\sum_{n \in \mathcal{S}} q^{n} \sum_{k \geq 0} \frac{q^{n k}}{(q ; q)_{k}} \sim d_{\mathcal{S}} \cdot \sum_{k \geq 1} \frac{q^{k}}{(q ; q)_{k}} .
$$

By the $q$-binomial theorem, the inner sum over $k \geq 0$ is equal to $\left(q^{n} ; q\right)_{\infty}^{-1}$, and the summation on the right equals $(q ; q)_{\infty}^{-1}-1$. Then multiplying both sides of $(3.18)$ by $(q ; q)_{\infty}$ implies by well-known generating function arguments that, as $q \rightarrow 1$, we have

$$
\sum_{n \in \mathcal{S}} q^{n}(q ; q)_{n-1}=\sum_{\lg (\lambda) \in \mathcal{S}} \mu_{\mathcal{P}}^{*}(\lambda) q^{|\lambda|} \sim d_{\mathcal{S}}
$$

Remark 3.8. Taken together, Theorems 3.6 and 3.7 present an "Alladi duality" connecting relations over minimal and maximal elements of multisets, as exemplified in [Al7\%, Al17, Wa3]. Whereas in [Al7y] the smallest and largest prime factors of $n$ induce a duality, here the duality is between the smallest and largest parts of $\lambda$.

\section{Proof of Theorem 1.3 and its corollaries}

\section{A. Partition convolution identities}

Now we turn our attention toward proving the partition convolution identities analogous to formulas of Wang [Wa3]. It is an instance of (2.12) for arbitrary partition-theoretic functions $a(\lambda), b(\lambda)$ that

$$
\left(\sum_{\lambda \in \mathcal{P}} a(\lambda) q^{|\lambda|}\right)\left(\sum_{\lambda \in \mathcal{P}} b(\lambda) q^{|\lambda|}\right)=\sum_{\lambda \in \mathcal{P}}(a * b)(\lambda) q^{|\lambda|}=\sum_{n \geq 0} \sum_{\operatorname{sm}(\lambda)=n}(a * b)(\lambda) q^{|\lambda|}
$$

where we now take $*$ to be partition-theoretic convolution (which is apparent from the context). We record a useful formula for the inner $\operatorname{sum} \sum_{\operatorname{sm}(\lambda)=n}$ of the right-hand double series. This will serve as another building block for further arithmetic density formulas.

Lemma 4.1. For $a(\lambda), b(\lambda)$ functions on $\mathcal{P}$, we have

$$
\begin{aligned}
\sum_{\operatorname{sm}(\lambda)=n}(a * b)(\lambda) q^{|\lambda|} & =\left(\sum_{\operatorname{sm}(\gamma)=n} a(\gamma) q^{|\gamma|}\right)\left(b(\emptyset)+\sum_{\operatorname{sm}(\gamma) \geq{ }^{*} n} b(\gamma) q^{|\gamma|}\right) \\
& +\left(a(\emptyset)+\sum_{\operatorname{sm}(\gamma) \geq^{*} n} a(\gamma) q^{|\gamma|}\right)\left(\sum_{\operatorname{sm}(\gamma)=n} b(\gamma) q^{|\gamma|}\right)
\end{aligned}
$$


where $\geq^{*}$ denotes $>$ if the associated summands vanish at partitions with any part repeated, and denotes $\geq$ otherwise.

Proof. The right-hand side of Lemma 4.1 generates the partitions $\lambda$ with smallest part $n$ on the left. That the summands on the left should have the form $q^{|\lambda|} \sum_{\delta \mid \lambda} a(\delta) b(\lambda / \delta)=\sum_{\delta \delta^{\prime}=\lambda}\left(a(\delta) q^{|\delta|} \cdot b\left(\delta^{\prime}\right) q^{\left|\delta^{\prime}\right|}\right)$ is obvious from standard partition generating function ideas, e.g. (4.20). Now consider $\lambda=\delta \delta^{\prime}$ on the left, choosing $\delta$ from a sum with coefficients $a(\gamma)$ on the right-hand side, and $\delta^{\prime}$ from a sum with coefficients $b(\gamma)$. If subpartition $\delta$ contains the copy of part $n$ satisfying the " $\operatorname{sm}(\lambda)=n$ " condition, then $\lambda$ is generated by the first term " $\left(\sum_{\mathrm{sm}(\gamma)=n}\right)\left(b+\sum_{\mathrm{sm}(\gamma) \geq{ }^{*} n}\right)$ " on the right: $\delta$ comes from the $\sum_{\mathrm{sm}(\gamma)=n}$ factor, and $\delta^{\prime}$ comes from the $b+\sum_{\mathrm{sm}(\gamma) \geq{ }^{*} n}$ factor. If, on the other hand, $\delta^{\prime}$ contains the designated smallest part $n$, then $\lambda$ is generated by the second term " $\left(a+\sum_{\mathrm{sm}(\gamma) \geq{ }^{*} n}\right)\left(\sum_{\mathrm{sm}(\gamma)=n}\right)$ " on the right: $\delta$ comes from $a+\sum_{\mathrm{sm}(\gamma) \geq{ }^{*} n}$ and $\delta^{\prime}$ comes from the $\sum_{\mathrm{sm}(\gamma)=n}$ factor.

We wish to find formulas with coefficients of the form $\mu_{\mathcal{P}} * a$, to yield Theorem 1.3. Now, for $|q|<1$, recall the $q$-bracket $\langle a\rangle_{q}:=\frac{\sum_{\lambda \in \mathcal{P}} a(\lambda) q^{|\lambda|}}{\sum_{\lambda \in \mathcal{P}} q^{|\lambda|}}=(q ; q)_{\infty} \sum_{\lambda \in \mathcal{P}} a(\lambda) q^{|\lambda|}$ of Bloch and Okounkov. The $q$-bracket can be interpreted as the expected value of $a(\lambda)$ over all partitions, given certain hypotheses from statistical physics; it is connected to modular, quasi-modular and $p$-adic modular forms (see [BlOk00, BOW, GJT16, Za15]). Then (4.20) gives

$$
\sum_{\lambda \in \mathcal{P}}\left(\mu_{\mathcal{P}} * a\right)(\lambda) q^{|\lambda|}=\langle a\rangle_{q}
$$

which of course leads to further partition convolution relations from $q$-bracket examples in the literature. Taken over all partitions $\lambda$, we see $\mu_{\mathcal{P}} * a$ induces a type of averaging phenomenon, as well as a connection to the theory of modular forms.

To restrict smallest parts to the subset $\mathcal{S}$, we require a specialization of Lemma 4.1.

Lemma 4.2. For a $(\lambda)$ a function on $\mathcal{P}$, we have

$$
\sum_{\operatorname{sm}(\lambda)=n}\left(\mu_{\mathcal{P}} * a\right)(\lambda) q^{|\lambda|}=\widetilde{A}_{n}(q) \cdot q^{n}\left(q^{n+1} ; q\right)_{\infty},
$$

where $\widetilde{A}_{n}(q):=A_{n}(q)-a(\emptyset)$ with $A_{n}(q)$ as in $(1.8)$.

Proof. Swap $a, b$ in Lemma 4.1, noting $a * b=b * a$, and let $b(\lambda)=\mu_{\mathcal{P}}(\lambda)$ to yield

$$
\begin{aligned}
\sum_{\operatorname{sm}(\lambda)=n}\left(\mu_{\mathcal{P}} * a\right)(\lambda) q^{|\lambda|}= & -q^{n}\left(q^{n+1} ; q\right)_{\infty}\left(a(\emptyset)+\sum_{\operatorname{sm}(\gamma) \geq^{*} n} a(\gamma) q^{|\gamma|}\right) \\
& +\left(q^{n+1} ; q\right)_{\infty}\left(\sum_{\operatorname{sm}(\gamma)=n} a(\gamma) q^{|\gamma|}\right) .
\end{aligned}
$$

Factor $q^{n}$ out of the $\sum_{\operatorname{sm}(\gamma)=n}$ factor on the right; the summands undergo the transformation $a(\gamma) q^{|\gamma|} \mapsto a(\gamma \cdot(n)) q^{|\gamma|}$. Then a little arithmetic gives

$$
\sum_{\operatorname{sm}(\lambda)=n}\left(\mu_{\mathcal{P}} * a\right)(\lambda) q^{|\lambda|}=q^{n}\left(q^{n+1} ; q\right)_{\infty}\left(a((n))-a(\emptyset)+\sum_{\operatorname{sm}(\gamma) \geq^{*} n}[a(\gamma \cdot(n))-a(\gamma)] q^{|\gamma|}\right) .
$$

The parenthetical term on the right is exactly $\widetilde{A}_{n}(q)$, completing the proof. 
This lemma works out nicely when the choice of $a(\lambda)$ allows one to factor out aspects of $\widetilde{A}_{n}(q)$, or simplify the sum otherwise. Now the following general lemma, wrapping together the preceding formulas, will lead to the proof of Theorem 1.3 as $q \rightarrow 1$.

Lemma 4.3. For $a(\lambda)$ a partition-theoretic function, $f(n)$ an arithmetic function, we have

$$
\sum_{\lambda \neq \emptyset}\left(\mu_{\mathcal{P}} * a\right)(\lambda) f(\operatorname{sm}(\lambda)) q^{|\lambda|}=(q ; q)_{\infty} \sum_{n \geq 1} \frac{f(n) \widetilde{A}_{n}(q) q^{n}}{(q ; q)_{n}},
$$

where $\widetilde{A}_{n}(q):=A_{n}(q)-a(\emptyset)$ with $A_{n}(q)$ as in (1.8).

Proof. Multiply Lemma 4.2 through by $f(n)$, and sum over $n \geq 1$, noting for each index $n$ the partitions generated on the left-hand side have smallest part $\operatorname{sm}(\lambda)=n$. Swapping order of summation on the left, then, takes $f(n) \mapsto f(\operatorname{sm}(\lambda))$. On the right-hand side, use the factorization $\left(q^{n+1} ; q\right)_{\infty}=$ $\frac{(q ; q)_{\infty}}{(q ; q)_{n}}$ to give the Lemma 4.3 .

\section{B. Proof of Theorem 1.3 and Corollaries 1.4 and 1.6}

We now have all the ingredients to prove Theorem 1.3 and its corollaries.

Proof of Theorem 1.3. Consider the identity in Lemma 4.3 above. By hypothesis we assume $a(\emptyset)=$ $1 .^{8}$ Letting $\chi_{\mathcal{S}}(n)$ denote the indicator function for $\mathcal{S} \subseteq \mathbb{N}$, make the substitution $f(n) \mapsto \frac{\chi_{\mathcal{S}}(n)}{f(n)}$ (hence the condition that $f$ not vanish on $\mathcal{S}$ in the denominator) to give

$$
\sum_{\operatorname{sm}(\lambda) \in \mathcal{S}} \frac{\left(\mu_{\mathcal{P}} * a\right)(\lambda)}{f(\operatorname{sm}(\lambda))} q^{|\lambda|}=(q ; q)_{\infty} \sum_{n \in \mathcal{S}} \frac{\widetilde{A}_{n}(q) q^{n}}{f(n) \cdot(q ; q)_{n}} .
$$

As $q \rightarrow 1$, if $A_{n}(q) \rightarrow 1$ for $n \in \mathcal{S}$, then $\widetilde{A}_{n}(q)=A_{n}(q)-1 \rightarrow 0$ in the summands on the right. Clearly the right side vanishes as $q \rightarrow 1$, which proves the first identity of the theorem.

Similarly, if $A_{n}(q) \rightarrow 1+f(n)$ for $n \in \mathcal{S}$, then $\widetilde{A}_{n}(q) \rightarrow f(n)$. In the limit, $\frac{\widetilde{A}_{n}(q)}{f(n)} \rightarrow 1$ and the right-hand side of (4.22) asymptotically equals $F_{\mathcal{S}}(q)$, which approaches $d_{\mathcal{S}}$ by Theorem 3.6. This is the content of the second identity and its analytic condition.

Remark 4.4. The idea is to set $f(n)=\lim _{q \rightarrow 1} \widetilde{A}_{n}(q)$ for $n \in \mathcal{S}$, so the right-hand side becomes $F_{\mathcal{S}}(q)$ in the limit.

Proof of Corollary 1.4. We have by hypothesis $A_{n}(q)=\widetilde{A}_{n}(q)+1 \rightarrow 0$, which is equivalent to having $\lim _{q \rightarrow 1} \widetilde{A}_{n}(q)=-1$. Then setting $f(k)=-1$ in $(4.22)$ gives the corollary as $q \rightarrow 1$.

Proof of Corollary 1.6. With $a, f, g$ and $b=b_{a, f, g}$ as defined in Corollary 1.6, following Wang's treatment, apply the partition Möbius inversion formula (2.11) to the definition of $b_{a, f, g}(\lambda)$ in the statement of the corollary to give

$$
\left(\mu_{\mathcal{P}} * a\right)(\lambda) \frac{g(\operatorname{sm}(\lambda))}{f(\operatorname{sm}(\lambda))}=\sum_{\delta \mid \lambda} \mu_{\mathcal{P}}(\delta) b_{a, f, g}(\lambda / \delta)=\left(\mu_{\mathcal{P}} * b\right)(\lambda) .
$$

Divide through by $g(\operatorname{sm}(\lambda))$ (thus the condition that it also is non-vanishing), multiply both sides by $q^{|\lambda|}$, sum over partitions $\lambda$ with $\operatorname{sm}(\lambda) \in \mathcal{S}$, then compare the result with the $A_{n}(q) \rightarrow 1+f(n)$ case of Theorem 1.3, to see

$$
\lim _{q \rightarrow 1} \sum_{\operatorname{sm}(\lambda) \in \mathcal{S}} \frac{\left(\mu_{\mathcal{P}} * b\right)(\lambda)}{g(\operatorname{sm}(\lambda))} q^{|\lambda|}=\lim _{q \rightarrow 1} \sum_{\operatorname{sm}(\lambda) \in \mathcal{S}} \frac{\left(\mu_{\mathcal{P}} * a\right)(\lambda)}{f(\operatorname{sm}(\lambda))} q^{|\lambda|}=d_{\mathcal{S}} .
$$

\footnotetext{
${ }^{8}$ A slightly more general theorem follows from using arbitrary $a(\emptyset)$ in this proof.
} 
Proof of Corollary 1.7. With $b(\lambda)=b_{a,-\varphi,-1}(\lambda), B_{n}(q)$ as defined in Corollary 1.7, if $B_{n}(q) \rightarrow 0$ on $\mathcal{S}$, the left-hand side of the resulting equation from (4.24) is the instance of Corollary 1.4 where $a$ is replaced by $b=b_{a,-\varphi,-1}$. The right-hand equality of (4.24) then yields Corollary 1.7.

\section{Further examples}

In this section we give further examples of applications of the formulas and methods developed above. We note in passing for any $q$-commensurate subset $\mathcal{S}$, that L'Hospital's rule yields infinitely many formulas for $\lim _{q \rightarrow 1} d_{\mathcal{S}}(q)$ by taking derivatives of the numerator and denominator of (3.14). Taking antiderivatives instead, then applying L'Hospital's rule, yields another arithmetic density relation for $q$-commensurate subset $\mathcal{S}$ :

$$
-\lim _{q \rightarrow 1} \frac{1}{\log (1-q)} \sum_{n \in \mathcal{S}} \frac{q^{n}}{n}=d_{\mathcal{S}}
$$

where $\log z$ denotes the principle branch of the complex logarithm function for $z \in \mathbb{C}$.

The asymptotic (3.16) is suggestive of further, less trivial formulas to compute $d_{\mathcal{S}}$.

Example 5.1. Let $\mu(k)$ denote the classical Möbius function, and let $f(k)$ be an arithmetic function that is not identically zero. For $\mathcal{S} \subseteq \mathbb{N}$ a q-commensurate subset, we have

$$
\lim _{q \rightarrow 1} \frac{\sum_{n \in \mathcal{S}} \sum_{k \geq 1}(\mu * f)(k) q^{n k}}{\sum_{k \geq 1} f(k) q^{k}}=d_{\mathcal{S}}
$$

where $*$ denotes classical Dirichlet convolution.

Proof. Take $q \mapsto q^{k}$ in (3.16). Multiply both sides of the asymptotic equality by $(\mu * f)(k)=$ $\sum_{d \mid k} \mu(d) f(k / d)$, sum over $k \geq 1$, and (noting the series are absolutely convergent) swap order of summation on the left. Then as $q \rightarrow 1$, well-known relations involving classical Lambert series and Dirichlet convolution give

$$
\sum_{n \in \mathcal{S}} \sum_{k \geq 1}(\mu * f)(k) q^{n k} \sim d_{\mathcal{S}} \cdot \sum_{k \geq 1} \frac{(\mu * f)(k) q^{k}}{1-q^{k}}=d_{\mathcal{S}} \cdot \sum_{k \geq 1} f(k) q^{k} .
$$

Dividing through by $\sum_{k \geq 1} f(k) q^{k}$ completes the proof.

Setting $f(k):=1$ for all $k \geq 1$, then Example 5.1 reduces to (3.15). Setting $f(k):=\sigma_{0}(k)$, the classical divisor function such that $\left(\mu * \sigma_{0}\right)(k)=1$, yields a Lambert series calculation.

Example 5.2. For $\mathcal{S} \subseteq \mathbb{N}$ a q-commensurate subset, we have

$$
\lim _{q \rightarrow 1} \frac{\sum_{n \in \mathcal{S}} \frac{q^{n}}{1-q^{n}}}{\sum_{n \geq 1} \frac{q^{n}}{1-q^{n}}}=d_{\mathcal{S}}
$$

Setting $f(1):=1, f(k):=0$ for $k>1$, viz. the function $f(k):=\sum_{d \mid k} \mu(d)$, gives another density formula that resembles a power series analogue of the first sum in (3.13).

Example 5.3. For $\mathcal{S} \subseteq \mathbb{N}$ a q-commensurate subset, we have

$$
\lim _{q \rightarrow 1} \sum_{n \in \mathcal{S}} \sum_{k \geq 1} \mu(k) q^{n k}=d_{\mathcal{S}}
$$


Setting $f(n)$ equal to the partition function $p(n)$ in Example 5.1, gives an example that looks more like the formulas in [OSW17] and the present work.

Example 5.4. Let $p(n)$ denote the partition function. For $\mathcal{S} \subseteq \mathbb{N}$ a q-commensurate subset, we have

$$
\lim _{q \rightarrow 1}(q ; q)_{\infty} \sum_{n \in \mathcal{S}} \sum_{k \geq 1}(\mu * p)(k) q^{n k}=d_{\mathcal{S}}
$$

Beyond giving analogues of classical convolution theorems as in Section 2. above, and giving formulas for expected value and arithmetic density, partition-theoretic convolution leads to other interesting $q$-series relations. Here is a nice consequence of Lemma 4.1, making another connection between partition convolution and modular forms.

Example 5.5. For $z \in \mathbb{C}, z \neq 0$, set $z^{\ell}(\lambda):=z^{\ell(\lambda)}, z^{-\ell}(\lambda):=z^{-\ell(\lambda)}$. Recall the rank generating function for strongly unimodal sequences, $U(z, q):=\sum_{n \geq 0} q^{n+1}(-z q ; q)_{n}\left(-z^{-1} q ; q\right)_{n}$. Then

$$
\sum_{\lambda \in \mathcal{P}}\left(z^{\ell} * z^{-\ell}\right)(\lambda) q^{|\lambda|}=1+\frac{z+z^{-1}}{(z q ; q)_{\infty}\left(z^{-1} q ; q\right)_{\infty}} U(-z, q) .
$$

Remark 5.6. We note in Example 5.5 a close relation to the crank generating function of AndrewsGarvan, $C(z, q)=\frac{(q ; q)_{\infty}}{(z q ; q)_{\infty}\left(z^{-1} q ; q\right)_{\infty}}($ see $[$ AnGa88]), viz.

$$
\sum_{\lambda \neq \emptyset}\left(z^{\ell} * z^{-\ell}\right)(\lambda) q^{|\lambda|}=\frac{\left(z+z^{-1}\right) C(z, q) U(-z, q)}{(q ; q)_{\infty}} .
$$

Proof. Take $a=z^{\ell}, b=z^{-\ell}$ in Lemma 4.1 to yield

$$
\begin{aligned}
\sum_{\operatorname{sm}(\lambda)=n}\left(z^{\ell} * z^{-\ell}\right)(\lambda) q^{|\lambda|} & =\frac{z q^{n}}{\left(z q^{n} ; q\right)_{\infty}} \cdot \frac{1}{\left(z^{-1} q^{n} ; q\right)_{\infty}}+\frac{1}{\left(z q^{n} ; q\right)_{\infty}} \cdot \frac{z^{-1} q^{n}}{\left(z^{-1} q^{n} ; q\right)_{\infty}} \\
& =\frac{z+z^{-1}}{(z q ; q)_{\infty}\left(z^{-1} q ; q\right)_{\infty}} \cdot q^{n}(z q ; q)_{n-1}\left(z^{-1} q ; q\right)_{n-1} .
\end{aligned}
$$

Summing over $n \geq 1$, then adding 1 to both sides of the equation to adjoin the $\lambda=\emptyset$ term on the left, and comparing with the definition of $U(z, q)$, completes the proof.

The rank generating function for strongly unimodal sequences is connected to mock modular and quantum modular forms (see e.g. [FOR13]). Example 5.5 itself, equal to $(z q ; q)_{\infty}^{-1}\left(z^{-1} q ; q\right)_{\infty}^{-1}$ by (4.20), is modular for $z$ a root of unity, up to multiplication by a rational power of $q$.

One may obtain different forms of partition convolution identities for $d_{\mathcal{S}}$ from those in Theorem 1.3 as consequences of Lemma 4.3, such as the following pair of identities.

Example 5.7. For $S \subseteq \mathbb{N}$ a q-commensurate subset, $a(\lambda)=\ell(\lambda)$ (partition length) and $\sigma_{0}(n):=$ $\sum_{d \mid n} 1$ as usual, we have

$$
\lim _{q \rightarrow 1} \frac{\sum_{\operatorname{sm}(\lambda) \in \mathcal{S}}\left(\mu_{\mathcal{P}} * \ell\right)(\lambda) q^{|\lambda|}}{\sum_{n \geq 1} \sigma_{0}(n) q^{n}}=d_{\mathcal{S}}
$$

For $S \subseteq \mathbb{N}$ a q-commensurate subset, $a(\lambda)=\operatorname{sz}(\lambda):=|\lambda|$ (partition size) and $\sigma_{1}(n):=\sum_{d \mid n} d$, we have

$$
\lim _{q \rightarrow 1} \frac{\sum_{\mathrm{sm}(\lambda) \in \mathcal{S}}\left(\mu_{\mathcal{P}} * \mathrm{sz}\right)(\lambda) q^{|\lambda|}}{\sum_{n \geq 1} \sigma_{1}(n) q^{n}}=d_{\mathcal{S}}
$$


Proof. For the first identity, set $a(\lambda)=\ell(\lambda)$ and $f$ equal to the indicator function of $\mathcal{S}$ in Corollary 4.3. Then since $\ell((n))=1, \ell(\emptyset)=0$, and $\ell(\gamma \cdot(n))-\ell(\gamma)=1$ for any $\gamma \in \mathcal{P}$, we have

$$
\widetilde{A}_{n}(q)=1+\sum_{\operatorname{sm}(\gamma) \geq n} q^{|\gamma|}=\frac{1}{\left(q^{n} ; q\right)_{\infty}}=\frac{(q ; q)_{n-1}}{(q ; q)_{\infty}} .
$$

Note from the right-hand side that $\widetilde{A}_{n}(q)$ does not satisfy either of the analytic conditions of Theorem 1.3 as $q \rightarrow 1$. However, by (5.28), it fits nicely into the right-hand side of Lemma 4.3:

$$
\sum_{\operatorname{sm}(\lambda) \in \mathcal{S}}\left(\mu_{\mathcal{P}} * \ell\right)(\lambda) q^{|\lambda|}=(q ; q)_{\infty} \sum_{n \in \mathcal{S}} \frac{\widetilde{A}_{n}(q) q^{n}}{(q ; q)_{n}}=\sum_{n \in \mathcal{S}} \frac{q^{n}(q ; q)_{n-1}}{(q ; q)_{n}}=\sum_{n \in \mathcal{S}} \frac{q^{n}}{1-q^{n}},
$$

which reduces the corollary to a rewriting of Example 5.2 above. Dividing through by $\sum_{n \geq 1} \frac{q^{n}}{1-q^{n}}=$ $\sum_{n \geq 1} \sigma_{0}(n) q^{n}$ and letting $q \rightarrow 1$ completes the proof of the first identity.

We prove the second identity along very similar lines, setting $a(\lambda)=\operatorname{sz}(\lambda)=|\lambda|$. Then since $|(n)|=n,|\emptyset|=0$, and $|\gamma \cdot(n)|-|\gamma|=n$ for any $\gamma \in \mathcal{P}$, in this case we compute

$$
\widetilde{A}_{n}(q)=n+\sum_{\operatorname{sm}(\gamma) \geq n} n q^{|\gamma|}=\frac{n}{\left(q^{n} ; q\right)_{\infty}}=\frac{n \cdot(q ; q)_{n-1}}{(q ; q)_{\infty}} .
$$

Again, this $\widetilde{A}_{n}(q)$ does not satisfy the conditions of Theorem 1.3; however, by (5.29), we have

$$
\sum_{\operatorname{sm}(\lambda) \in \mathcal{S}}\left(\mu_{\mathcal{P}} * \mathrm{sz}\right)(\lambda) q^{|\lambda|}=(q ; q)_{\infty} \sum_{n \in \mathcal{S}} \frac{\widetilde{A}_{n}(q) q^{n}}{(q ; q)_{n}}=\sum_{n \in \mathcal{S}} \frac{n q^{n}(q ; q)_{n-1}}{(q ; q)_{n}}=\sum_{n \in \mathcal{S}} \frac{n q^{n}}{1-q^{n}}
$$

Now, the right-hand side of these equalities can be rewritten

$$
\sum_{n \in \mathcal{S}} \frac{n q^{n}}{1-q^{n}}=\sum_{n \in \mathcal{S}} n \sum_{k \geq 1} q^{n k}=\sum_{k \geq 1} \sum_{n \in \mathcal{S}} n q^{n k}
$$

It follows from (3.16) that

$$
\sum_{n \in \mathcal{S}} n q^{n}=q \frac{d}{d q} \sum_{n \in \mathcal{S}} q^{n} \sim d_{\mathcal{S}} \cdot \frac{q}{(1-q)^{2}}
$$

which, combined with (5.30) above, gives

$$
\sum_{n \in \mathcal{S}} \frac{n q^{n}}{1-q^{n}} \sim d_{\mathcal{S}} \cdot \sum_{k \geq 1} \frac{q^{k}}{\left(1-q^{k}\right)^{2}}=d_{\mathcal{S}} \cdot \sum_{k \geq 1} \frac{k q^{k}}{1-q^{k}}=d_{\mathcal{S}} \cdot \sum_{k \geq 1} \sigma_{1}(k) q^{k},
$$

using well-known Lambert series identities in the right-hand equalities. Dividing through by $\sum_{n \geq 1} \sigma_{1}(n) q^{n}$ and letting $q \rightarrow 1$ gives the second identity.

Remark 5.8. Note that $\ell(\lambda)$ and $\mathrm{sz}(\lambda)$ are the $r=0$ and $r=1$ cases, respectively, of

$$
\mathrm{sz}_{r}(\lambda):=\sum_{\lambda_{i} \in \lambda} \lambda_{i}^{r}
$$

where the sum is over the parts $\lambda_{i} \in \mathbb{N}$ of partition $\lambda .{ }^{9}$ More generally, inserting $\operatorname{sz}_{r}(\lambda)$ for any integer $r \geq 0$ in place of $\ell(\lambda)$ and $\mathrm{sz}(\lambda)$, the steps of the proof of Example 5.7 continue to hold, but yielding $\left(\mu_{\mathcal{P}} * \mathrm{sz}_{r}\right)(\lambda)$ in the numerator and $\sigma_{r}(n):=\sum_{d \mid n} d^{r}$ in the denominator.

\footnotetext{
${ }^{9}$ One might write $\mathrm{sz}_{r}(\lambda)=|\lambda|_{r}$ to generalize the standard absolute value notation for partition size.
} 
Remark 5.9. Note from (5.31) and from the fact $\frac{q}{(1-q)^{2}}=\sum_{k \geq 1} \frac{\varphi(k) q^{k}}{1-q^{k}}=\sum_{k \geq 1} k q^{k}$, that

$$
\lim _{q \rightarrow 1} \frac{\sum_{n \in \mathcal{S}} n q^{n}}{\sum_{n \geq 1} n q^{n}}=d_{\mathcal{S}}
$$

which can also be found by applying L'Hospital's rule to (3.14) as $q \rightarrow 1$, as noted above.

We anticipate that formulas similar to those proved here will hold for sums over partitions with parts restricted in other ways to subsets $\mathcal{S}$ such as in Corollary 3.7, as well as for $q \rightarrow \zeta$ a root of unity not equal to one, along the lines of those in [OSW17]; but pursuing this was outside of our present aims. Using limiting techniques such as the Hardy-Ramanujan circle method [HR1918], techniques of Watson [Wat37] and other methods from the universe of $q$-series and modular forms - as well as limiting values of special functions at roots of unity - perhaps closed formulas can be computed for $d_{\mathcal{S}}$ beyond the cases of $\mathcal{S}$ being integers in arithmetic progressions, $k$ th power-free integers, and other subsets of $\mathbb{N}$ with arithmetic densities already explicitly known.

Acknowledgement. We wish to express our gratitude to A.V. Sills for computing the values in Table 2, and to Paul Pollack for providing advice and references on Tauberian theorems. Moreover, the second author thanks G.E. Andrews and Prof. Sills for discussions on convergence, divergence and $q$-analogues that strongly informed this work.

\section{References}

[Al77] K. Alladi, Duality between prime factors and an application to the prime number theorem for arithmetic progressions, J. Numb. Th., 9, 436-451, 1977.

[Al17] K. Alladi, A duality between the largest and smallest prime factors via the Möbius function and arithmetical consequences, Emory University Number Theory Seminar, February 28, 2017.

[AlEr77] K. Alladi and P. Erdös, On an additive arithmetic function, Pacific J. Math. 71.2 (1977): $275-294$.

[An98] G.E. Andrews, The theory of partitions, no. 2, Cambridge University Press, 1998.

[AnGa88] G.E. Andrews and F.G. Garvan, Dyson's crank of a partition, Bulletin of the American Mathematical Society 18.2 (1988): 167-171.

[AnSt73] G.E. Andrews and A. Stenger, Solutions to Advanced Problems \# 5865, Amer. Math. Monthly 80 (1973), $1147-1148$.

[BlOk00] S. Bloch and A. Okounkov, The character of the infinite wedge representation, Adv. Math. 149 (2000), no. 1, 1-60.

[BOW] K. Bringmann, K. Ono, and Ian Wagner, Eichler integrals of Eisenstein series as q-brackets of weighted t-hook functions on partitions, preprint.

[Da17] M. Locus Dawsey, A new formula for Chebotarev densities, Res. Numb. Th. 3 (2017) Art. 27.

[Fi88] N.J. Fine, Basic hypergeometric series and applications, Math. Surveys and Monographs 27, Amer. Math. Soc., Providence, 1988.

[FOR13] A. Folsom, K. Ono, and R.C. Rhoades, Mock theta functions and quantum modular forms, Forum of mathematics, Pi, Vol. 1, Cambridge University Press, 2013.

[Fr1880] G. Frobenius, Über die Leibnitzsche Reihe, Journal für die reine und angewandte Mathematik (Crelle's Journal) 89 (1880): 262-264.

[GJT16] M. Griffin, M. Jameson and S. Trebat-Leder, On p-adic modular forms and the Bloch-Okounkov theorem, Research in the Mathematical Sciences 3.1 (2016): 11.

[Ha38] G.H. Hardy, A Course of Pure Mathematics, 1938.

[HR1918] G.H. Hardy and S. Ramanujan, Asymptotic formulae in combinatorial analysis, Proceedings of the London Mathematical Society 17: 75-115, 1918.

[Ko] J. Korevaar, A century of complex Tauberian theory, Bulletin of the American Mathematical Society 39.4 (2002): $475-531$.

[KMS] M. Kural, V. McDonald, and A. Sah, Möbius formulas for densities of sets of prime ideals, Archiv. Math., in press.

[ORS17] K. Ono, L. Rolen and R. Schneider, Explorations in the theory of partition zeta functions, Exploring the Riemann Zeta Function, 190 years from Riemann's Birth, Springer, Editors: H. Montgomery, A. Nikeghbali, and M. Rassias, Springer, 2017, 223-264. 
[OSW17] K. Ono, R. Schneider, and I. Wagner, Partition-theoretic formulas for arithmetic densities, Analytic Number Theory, modular forms and q-hypergeometric series, Springer Proc. Math. Stat. 221 (2017), 611-624.

[Sc16] R. Schneider, Partition zeta functions, Research in Number Theory 2.1 (2016): 9.

[Sc17] R. Schneider, Arithmetic of partitions and the q-bracket operator, Proc. Amer. Math. Soc. 145.5 (2017): 1953-1968.

[Sc18] R. Schneider, Eulerian Series, Zeta Functions and the Arithmetic of Partitions, Ph.D. thesis, Emory University, 2018.

[Sc] R. Schneider, Partition-theoretic Abelian theorems, preprint.

[ScSi20] R. Schneider, and A.V. Sills. Analysis and combinatorics of partition zeta functions. International Journal of Number Theory (2020): 1-10.

[SwWo19] N. Sweeting and K. Woo, Formulas for Chebotarev densities of Galois extensions of number fields, Res. Numb. Th. 5 (2019) Art. 4.

[Wa1] B. Wang, An analogue of a formula for Chebotarev densities, Int. J. Numb. Th., in press.

[Wa2] B. Wang, Ramanujan sum and Chebotarev densities, Ramanujan J., in press.

[Wa3] B. Wang, Analogues of Alladi's formula, preprint.

[Wat37] G.N. Watson, The mock theta functions (2), Proceedings of the London Mathematical Society 2.1 (1937): $274-304$.

[Za15] D. Zagier, Partitions, quasimodular forms, and the Bloch-Okounkov theorem, The Ramanujan Journal (2015): 1-24.

\section{Ken Ono}

Department of Mathematics

University of Virginia

Charlottesville, VA 22904, USA

e-mail: ken.ono691@virginia.edu

\section{Robert Schneider}

Department of Mathematics

University of Georgia

Athens, GA 30602, USA

e-mail: robert.schneider@uga.edu

\section{Ian Wagner}

Department of Mathematics

Vanderbilt University

Nashville, TN 37240, USA

e-mail: ian.c.wagner@vanderbilt.edu 